\title{
REAL-TIME GIS AND ITS APPLICATION IN INDOOR FIRE DISASTER
}

\author{
Weiping $\mathrm{Xu}^{\mathrm{a}}{ }^{*}$, Qing Zhu ${ }^{\mathrm{a}}$, Yeting Zhang ${ }^{\mathrm{a}}$, Yulin Ding ${ }^{\mathrm{a}}$, Mingyuan $\mathrm{Hu}^{\mathrm{b}}$ \\ ${ }^{\text {a }}$ State Key Laboratory of Information Engineering in Surveying, Mapping and Remote Sensing, Wuhan University, \\ China - yuqiexing@gmail.com, (zhuqing, zhangyeting)@263.net, rainforests@126.com \\ ${ }^{\mathrm{b}}$ Institute of Space and Earth Information Science, The Chinese University of Hong Kong, Hong Kong - \\ humingyuan@gmail.com
}

KEY WORDS: Real-time GIS, Spatiotemporal Data Model, Indoor Fire Disaster

\begin{abstract}
:
GIS is now faced with the challenge how to represent and understand the fast-paced, constantly changing world, given increasingly real-time data including readings from large-scale distributed sensors and large quantities of simulation data generated by computation models. Traditional static GIS pays more attention to representing historic data and Temporal GIS(TGIS) only treats time as a occasional but not critical factor and can't support the explicit change representation. In this context, Real-time GIS(RGIS) is put forward and its data model becomes a key problem how to make an appropriate abstraction over the rapid changes implied in real-time data stream and establish the general interaction relationship between them. The paper proposes a spatiotemporal changeoriented three-domain model with the emphasis on the semantic interaction relationship of object, event and process. Based on this model, a semantic enrichment method for multi-scale spatiotemporal change is put forward. Finally, as a RGIS application, indoor fire disaster simulation is illustrated and proves the validity of the proposed model.
\end{abstract}

\section{INTRODUCTION}

After the start of the 21st century, a variety of new sensors have been globally deployed to collect real-time data in service to earth observation networks, social networks, Internet of things and so on(Aggarwal, 2011, ESRI, 2010, Li, 2009). Meanwhile, the significant advances in computer technologies have greatly contributed to the development of model-based decision support for handling complex environmental, engineering, industrial, economical and social problems(Washington et al., 2009), which would generate lots of simulation data dynamically(Hey et al., 2009). As a result, the challenge emerges how to make good use of such accessible but overwhelming data stream in tracking and understanding the real-world changes especially when it exceeds our capacity to validate, analyse, visualize, store, and curate the information(Collins, 2010). For example, in increasingly frequent natural hazards and man-made accidents, given large quantities of on-site monitoring data from wireless sensor network and off-site computation results from environmental simulators, it is an urgent demand for governments and institutes to provide more capable tools and valid guidance for rapid-accurate emergency detection, management, prediction and response(Kwan et al., 2005, Laurini et al., 2005).

In the recent development of information science, DataIntensive scientific discovery, known as the fourth paradigm taking place of Computational scientific discovery, is becoming a hot research topic(Hey et al., 2009). Similarly, GIS as a powerful tool of geospatial information science is expected to be adapted to all these real-time data involvement and datadriven application possibilities. Traditional GIS method aims to map the "snapshot" of geographical world in a moment into computer databases by geospatial data persistence, followed by further development and integration of on-demand application functions operated on "out-dated" database records. However, even the "current" snapshot falls out of sync with fast-paced, constantly changing world(Artz, 2013). More specifically, its long-term focus on relatively static mode of analysing historic data, needs to be shifted to a far more dynamic process of monitoring, simulation and decision making, in which context RGIS is put forward as one potential development in the future of GIS (Goodchild, 2011). For example, GeoEvent Processor is a dynamic platform developed by ESRI for satisfying RGIS applications(ESRI, 2013). It gives users the ability to connect to real-time data streams from a wide variety of sensors, perform continuous processing and analysis of those data streams, and send relevant information to users or other systems. It can be configured to receive real-time weather and pollution measurements from a network of sensors and trigger alarms when specific pollution or wind thresholds are met. Or it can be used to detect and highlight vehicles that are speeding, stopped for a long period of time, or moving away from a predefined route. A general running framework for RGIS platform is illustrated in Figure 1. After real-time accessing module receives continuous data stream, online processing module will establish its spatiotemporal registration and correlation. Change monitoring module is used to extract the change information from real-time data which can support further simulation analysis. Their results can be directly sent by push centre to users. Register centre is for registration of real-time data type and module ability to be known by system. The data management module based on multi-level cache and distributed storage is necessary to support the above modules and dynamic visualization.

\footnotetext{
* Corresponding author.
} 


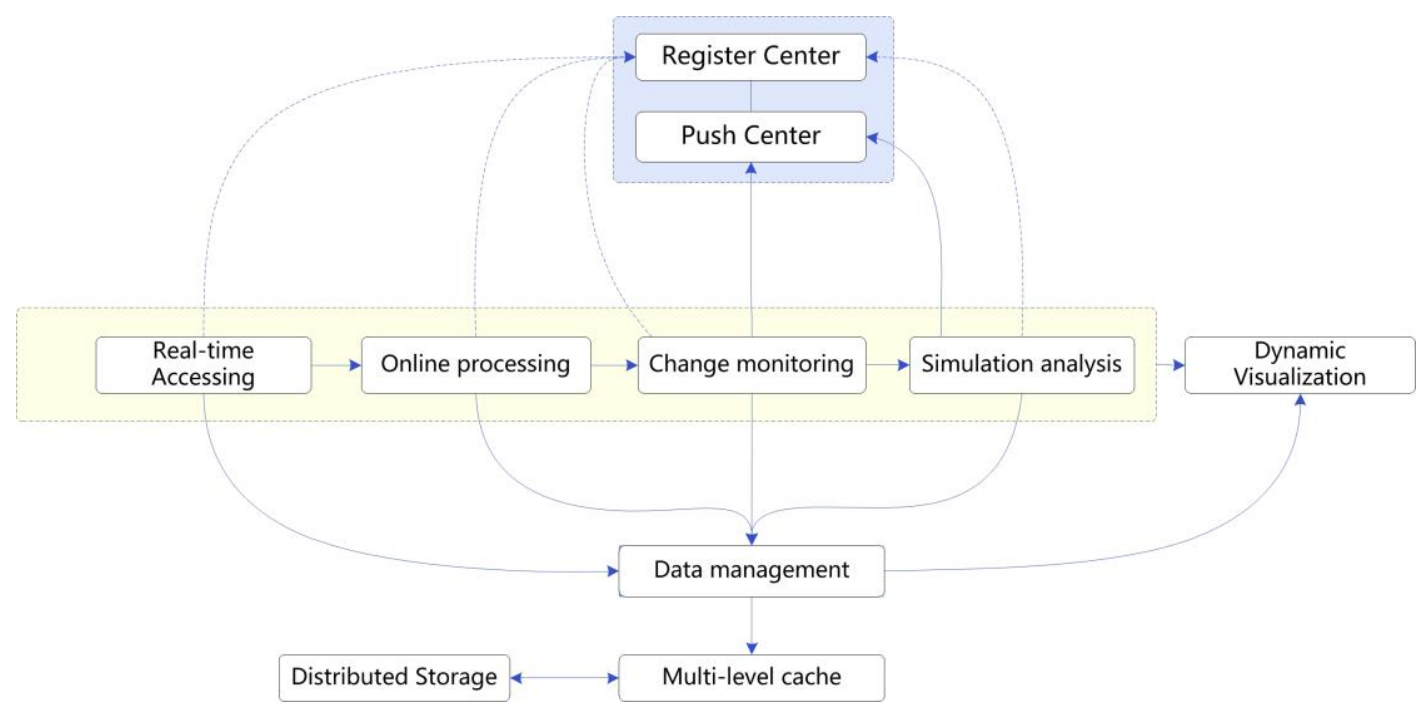

Figure 1. General running framework for RGIS platform

For effective representation of time-varying geospatial data, spatiotemporal data model has been one key research of TGIS in decades. TGIS is expected to monitor and analyse successive states of spatial entities, and also be equipped to study dependencies between linked entities(Claramunt et al., 1995). Therefore, existing TGIS data models mainly focus on recording the apparent states of multi-variable "things" involved in a change by linear timestamps and representing the implicit "happening" by the link of states before and after a change happens(Worboys, 2005). The temporal and spatial facts it records can be used to analyse spatiotemporal change patterns and infer underlying processes and relationship when needed(Claramunt et al., 1998). However, this framework can not support any deeper investigation into changes without explicit details on how and why the states vary in certain order and how the order is influenced especially in continuously changing geographical phenomenon. It is also explained from the above why most of TGIS data models are suitable for tracking such changes as land-use redistribution and object movement(Pelekis et al., 2004, Worboys, 2005), which seems to happen so "slowly" or "occasionally" that people only need to lay importance to discrete consequences instead of their intrinsic mechanism or dynamics (In fact, most changes are often observed without knowledge about the mechanisms). But for RGIS which treats time as a critical factor, any real-time change information are worth to detect and perceive in support of dynamic simulation and in-time forecast. In conclusion, how to make an appropriate abstraction over the rapid changes implied in real-time data stream and establish the general interaction relationship between them becomes a key problem of RGIS. It will be paid much attention to in the Section 2 and 3 of the paper.

Indoor fire disaster characterized by incident abruptness and process variability, is one of most serious and complex disasters in daily life (Hottel, 1984). Especially with the trend of composite indoor space and comprehensive architectural functions applied in high-rise building, underground shopping mall, hospital, museum and airport, indoor structure and facility is becoming so complicated that traditional methods used in indoor navigation and emergency evacuation face more difficulties in indoor fire safety project(Rüppel et al., 2010). This can be explained from two aspects. Firstly in the initial fire growth, the change of sensitive environment elements like smoke spread and heat transfer is regarded to be mainly affected by enclosure geometry, space connectivity and surrounding object's properties like its type, amount, position, spacing, orientation, and surface area of the fuel packages(Karlsson et al., 2000). The complicated inner structure will make the generation and movement of these highly dynamic elements more unpredictable even by the rigid fire model analysis. Secondly, traditional building information model will treat the whole spatial structure as static features to construct a geometric topological network for evacuation path analysis but ignore the fact that the function, state and lifetime of building components will undergo changes at any time in fire scene(Isikdag et al., 2008). For example, the enablement of fire roll shutter will block the original connected spaces; the breakage of window by heat will affect the ventilation conditions. In conclusion, decision makers have to shift their focus on the macro-scale response to detailed prediction of fire spread and improve its accuracy by accessing real-time data. The Section 4 will illustrate how RGIS data model can be used for indoor fire representation.

\section{SPATIOTEMPORAL REPRESENTATION TO SUPPORT REAL-TIME GIS}

The representation and querying method of spatiotemporal changes is the basis of a spatiotemporal data model. From an ontological perspective of change, all that exists can be basically divided into continuants and occurrents. A continuant exists over a period of time in which it is wholly present at any moment, and during its lifetime it may endure changes and possess different properties at different times; an occurrent cannot undergo any change and is not wholly present at any time short of its entire duration(Grenon et al., 2004). There is no doubt that RGIS should support the spatiotemporal representation of both of them and their relationship. Three requirements are listed as follows:

\subsection{Queries about involved continuants in space, time and} theme

In GIS, the state of an object (continuant) at a time point is represented by its spatial occupation and non-spatial attributes known as thematic. So the basic queries GIS support can be 
categorized into three: queries about attributes of entities; queries about locations, spatial properties, and spatial relationships; queries about time, temporal properties and temporal relationships(Yuan, 1999). Given the morphology, topology and attributes of a spatiotemporal object, eight types of change is allowed(Pelekis et al., 2004). For example, the snapshot model can answer simple temporal queries from the perspective of cartography but its capability to support complex spatial-temporal queries is limited(Langran et al., 1988). And the analysis would be made difficult to detect changes and obtain related change data about its magnitude, influence, trend and so on. The three-domain model represents semantics, space and time separately and provides links between them to describe geographic changes(Yuan, 1999), but the links between involved continuants would be very complex in the representation of multi-variable changes.

\subsection{Queries about occurrents based on application level}

Occurrents are known as things that happen to continuants. They may represent abrupt change or can be triggered when gradual evolution is considered to be significant enough (over some predefined threshold) to register change. An event is defined as an application-driven concept that supports a cognitive interpretation of a significant pattern of change(Claramunt et al., 2000). Event-oriented spatiotemporal data model (ESTDM) is used to represent the events explicitly which stores changes in relation to a previous state rather than a snapshot of an instance(Peuquet et al., 1995). Most of the time these events do not occur at random because they are constrained to previous status and obey to underlying evolution mechanism which is illustrated as process. How to represent and store process information, which provides advantages for querying and understanding processes is what the process-based data model focuses on(Reitsma et al., 2005). The hierarchical representation of changes based on the application level is necessary to describe change development at different spatial and temporal granularities. The common spatiotemporal hierarchy like "Event-Process-State" and "Event-ProcessSequence-Region" just extract simple processes from discrete temporal information and group them as an event(Yuan, 2001, Yuan et al., 2003).

\subsection{Queries about influences and causes between occurrents}

Modelling a change is not merely recording and storing itself. In RGIS simulation and analysis, insights may be gained into how to describe the interaction of changes which is important to determining how various processes propagate over time and how to reflect distinct spatiotemporal evolution characteristics during separate stages, under multiple influences and driven by different causes in applications. For example, the analysis of disaster chains focuses more on the causal relations of successive disasters.

\section{SPATIOTEMPORAL CHANGE-ORIENTED THREE- DOMAIN MODEL}

\subsection{Spatiotemporal Change-oriented Conceptual Model}

TGIS can relate the spatial components, the temporal components and thematic components with objects to represent object-oriented changes. The proposed model attaches more importance to the fact that the advance of time brings out the spatial change and thematic change. Therefore, it is necessary to make the abstraction over the time domain into the changedriven force with the integration of space and theme domain into the change pattern, which is regarded as spatiotemporal change-oriented data model. The proposed model is established through the links of change factor, change scale and change semantics as shown in Figure 2.

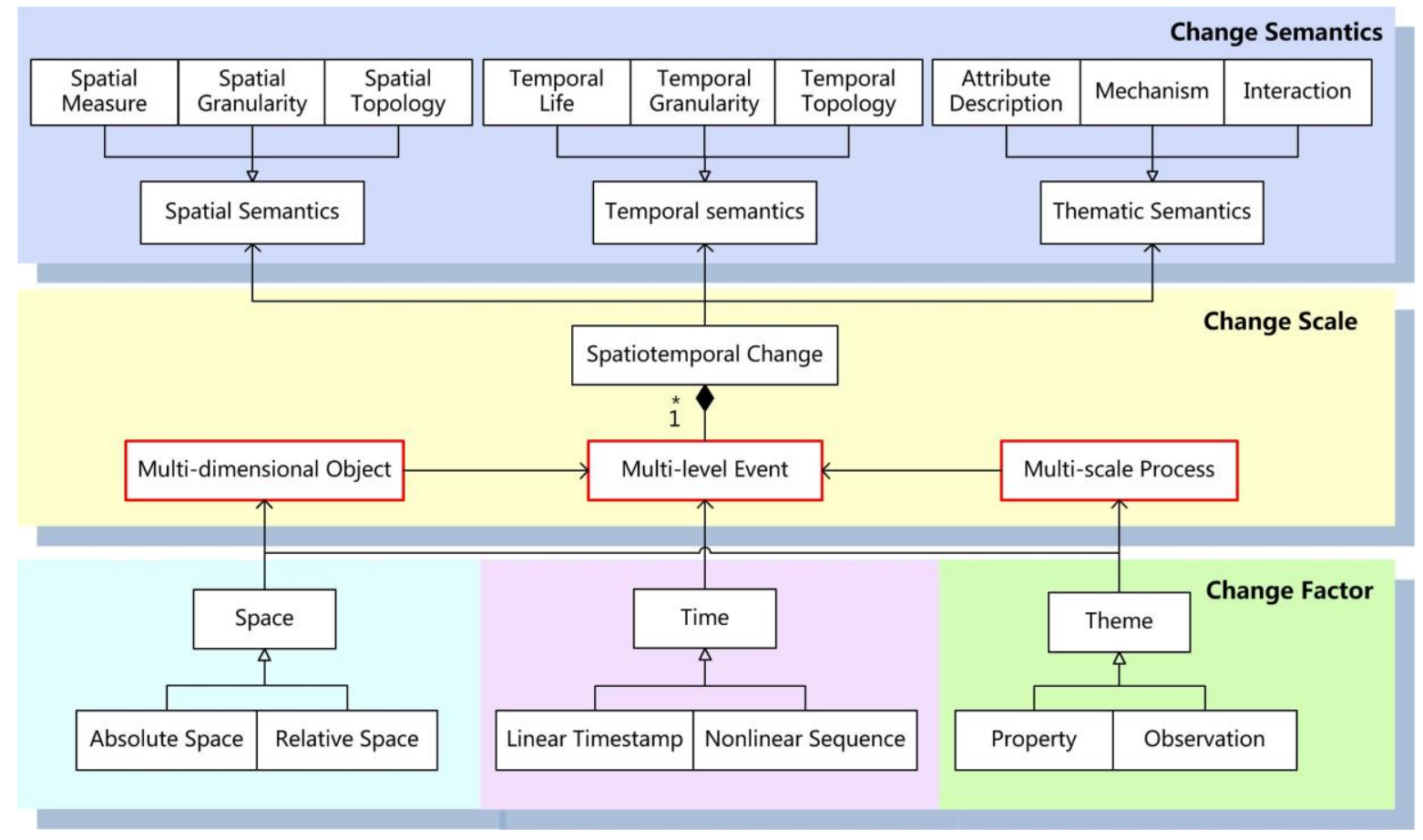

Figure 2. Spatiotemporal Change-oriented Conceptual Model 


\section{CHANGE SCALE}

The appropriate modeling of the real-world changes is determined by spatiotemporal scale, which reflects the abstraction of the change details from different understanding levels. The proposed model uses the interaction of three domains including object event-process to represent multi-scale changes as shown in following equation (1).

$$
C(s)=f_{c}(O, E, P)
$$

where $C(s)$ is a change in certain scale, $O$ means involved objects regarded as multi-dimensional, $E$ points to a set of defined events which can be divided into multi-level events according to the change scale, $P$ stands for the physical process which can include sub processes.

Equation (2) gives detailed definition of these three domains. Here, $O$ is thought to be possessed with spatial components $S$ and thematic components $T$. Each $P$ is governed by a process model $M(t)$ as its main body which is often expressed in the form of mathematical equations or functions. There is no doubt that it at least has one parameter: driven time $t . \overrightarrow{P i}$ and $\overrightarrow{P O}$ are separately the input list and output list of variables needed by $M$. E provides a comprehensive context for the changes in similar pattern. Some events may be required to assign the initial values to input parameters when it is triggered such as sensor-detected events. The trigger condition $\overrightarrow{\text { Cond }}$ is often expressed as a predefined threshold to reach or equation to judge. Other events may impose constraints $\overrightarrow{\mathrm{Cons}}$ on the objects or the processes involved in their occurrence. For example, the event may limit the possible range of variable change or make the process develop in another trend. Besides, $E$ has to define how the process model's input and output is linked with the representation of object and process, i.e., $f_{p}$ and $f_{o}$. Therefore, the spatiotemporal change can be represented as the aggregation of events which is related to processes and objects.

$$
\begin{aligned}
& O=f_{o}(S, T) \\
& P=f_{p}(M(t), \overrightarrow{P i}, \overrightarrow{P o}) \\
& E=f_{e}\left(\overrightarrow{\text { Cons }}, \overrightarrow{\text { Cond }}, f_{p}, f_{o}\right)
\end{aligned}
$$

\section{CHANGE FACTOR}

The change factor is more related to the spatiotemporal data which provide the basis for change modeling. The paper continues to define it from the perspective of space, time and theme components. The space occupation for an object can be represented by either absolute space or relation space. The absolute approach identifies space as a collection of points such as fields, while the relative approach focuses on real-world entities like vectors composed of point, line, surface and volume. The time factor can be basically divided into linear timestamp and nonlinear sequence. The former is still used in the representation of discrete status, while the latter for continuous analytic expressions. The thematic attributes include the intrinsic property and real-time observation. Common physical properties or behaviours which will not endure any change is thought to be intrinsic, real-time observation is mainly from sensors or simulators.

\section{CHANGE SEMANTICS}

The change semantics are represented through spatial semantics including spatial measure, spatial granularity and spatial topology; temporal semantics including temporal lifespan, temporal granularity, and temporal topology; thematic semantics including attribute description, mechanism and interaction. Here, the attribute description is more like a metadata of changing factors like variables, mechanism is a meaning given by the process model, and the interaction is between the object, event and process, which will be researched in next section.

\subsection{Semantic Interaction Relationship between Object- Event- Process}

In order to support the representation of evolution characteristics during separate stages, under multiple influences and driven by different causes, the elementary interaction relationships between object-event-process are defined as follows (Figure 3):

Description: A descriptive event contains the context of inner process and can execute influence on other outer processes.

Feedback: A feedback event doesn't relate with any inner process and can't execute any effect on other processes, but records some typical characteristics of the process evolution. People often separate a whole change into several meaningful stages from the temporal view according to these characteristics. If the spatiotemporal scale was small enough, each spatiotemporal unit could be recognized as a feedback event.

Influence: An influential event can execute an effect on other processes, but its inner process can be ignored as "virtual process". For example, detected events reported by sensors fall into this type. How the event is triggered by some change and detected by sensors can be given no consideration but how it influences other changes is important. It can be further classified into composite event and primitive event. The former is a measurable change in the attribute values of a sensor data sequence while the latter is composed of them by logical operators and spatiotemporal operators.

Cause: A casual event is regarded as the direct cause of other processes, including initiation and termination cause. It can be an influent or descriptive event.

Involvement: The local changes of involved objects will be constrained by the event.

Action: The object acts in the process as a principal part, and its status change is determined by the process model. 
Join: The object joins the process as a necessary part but its status may not be changed. It is used by the process model to calculate action objects' status.

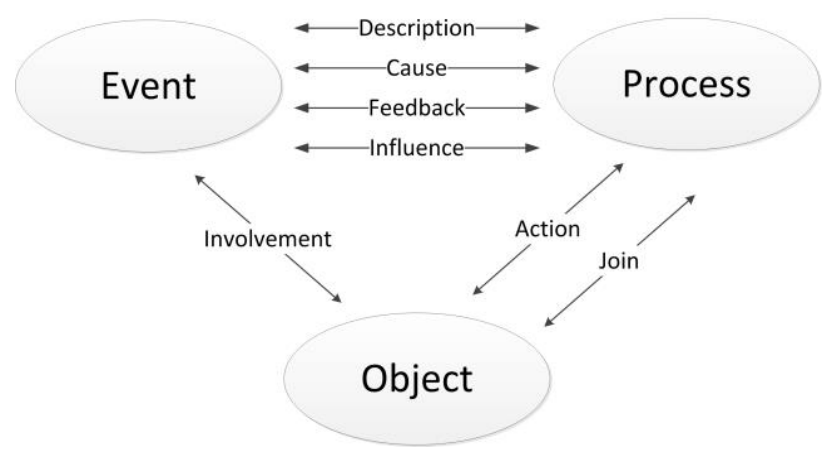

Figure 3. Semantic interaction relationship between ObjectEvent- Process

\subsection{Semantic Enrichment Method for Multi-scale Spatiotemporal Change}

According to the semantic interaction relationship between object-event-process, the semantic enrichment for multi-scale changes can be implemented using the event-based and processbased method, which is illustrated in Figure 4. In event-based representation, each event contains sub events in the lower level, which describes the happening context for inner process joined by the objects; while in process-based method, each process can be thought to be composed of sub processes, which is related with casual/influential/feedback events involved with the objects. The two methods can be related with each other to form a complete semantic description to satisfy the queries about the influences and causes between changes. For example, when inner process is queried using the event-based method, its sub processes and related events can be queried using the processbased method. It is worthy to mention the involved objects in the two methods may also be multi-scale. Based on these two methods and their relationship, the change description can be established.

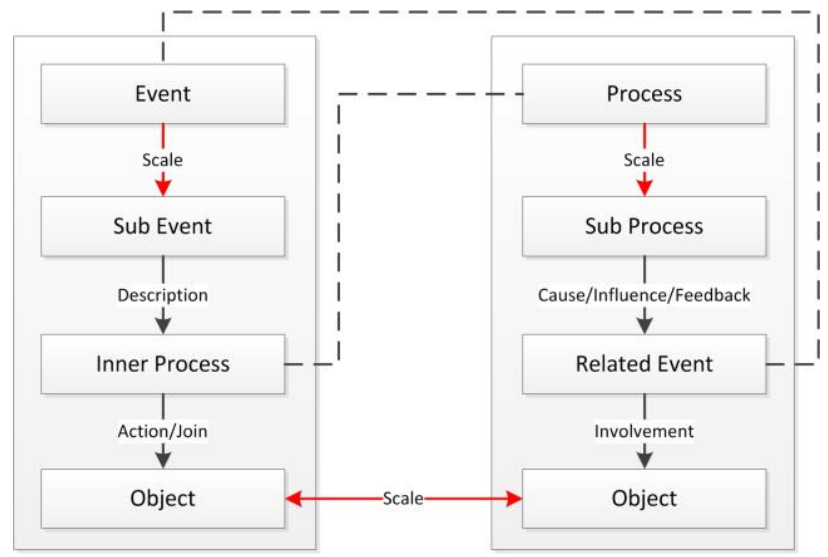

Figure 4. Semantic enrichment method for multi-scale spatiotemporal change

\section{REAL-TIME GIS CASE: INDOOR FIRE DISASTER SIMULATION}

In this paper, RGIS can be used for indoor fire simulation in following aspects.

\subsection{Fire Detection}

The ignition can be detected at once by composite monitoring from multi-source sensors. For example, video-based fire detection(VFD) is more employed because videos can 'see' a fire from a long distance without the smoke or heat needing to get to the detector first(Töreyin et al., 2006), and then other sensors can be used to measure environmental elements around the video-monitoring area such as smoke and flame in case of false alarms from VFD. So the fire-detection event as a composite event is regarded as composed of several primitive events. The logical operator <AND> is used because all of them have to be assured to happen and spatiotemporal operator $<$ BEFORE > means they should happen in order or else at the same time.

[VFD]

$<$ AND $><$ BEFORE $>$

[Flame-sensor Detection] $<$ AND $>$

[Smoke-sensor Detection]

Here, the variables of video, flame wavelength and smoke intensity are firstly registered by real-time accessing module into the register center. The detection algorithm are separately registered by change monitoring module. The size and location of the ignition source and its property like heat release rate can be extracted from the detected information to drive the fire simulation.

\subsection{Representation of Fire Spread}

Fire spread is the main process to be described in indoor fire simulation. In a smaller scale, it can be further divided into sub processes including solid phase pyrolysis, heat transfer, momentum transport and gas phase combustion. Pyrolysis is the decomposition or transformation of a compound caused by heat that produce the gaseous fuel which is the first chemical reaction that occurs in the burning of many solid fuels, like wood, cloth, paper, and plastic. So ignition event can be thought of the direct cause of the pyrolysis, which process can be ignored as virtual and only influenced by the fire-detection event.

Flashover is a very rapid and sudden transition from a growing fire to a fully developed fire, where all the combustible material in the enclosure is ignited, because all exposed surfaces are heated to the ignition temperature and emit flammable gases as a result of radiation from hot layer which threats the life of firefighters and rescue work. It can be judged as a feedback event for fire spread process when the Upper Layer Temperature reaches $600{ }^{\circ} \mathrm{C}$.

In the indoor fire disaster, some events affecting the fire development may happen in a sudden. For example the enablement of fire roll shutter will block the original connected spaces and affect the momentum transport; the breakage of window by heat will affect the ventilation conditions; sprinkles will automatically spray to the fire area to control the fire development. All these events can be regarded as influential events.

The typical semantic interactions between the above changes in indoor fire disaster using RGIS data model are listed in Table 1. 


\begin{tabular}{|lllll|}
\hline Change A & Change domain & Change B & Change domain & Semantic Interaction \\
\hline Indoor Fire & <Event> & Fire Spread & <Process> & Description \\
Flashover & <Event> & Fire Spread & <Process> & Feedback \\
Ignition & <Event $>$ & Pyrolysis & <Process> & Cause \\
Fire Detection & <Event $>$ & Ignition(virtual) & <Process> & Influence \\
Sprinkle Spray & $<$ Event $>$ & Heat Transfer & <Process> & Influence \\
Shutter Block & <Event $>$ & Momentum Transport & <Process> & Influence \\
Building & $<$ Object> & Indoor Fire & <Event> & Involvement \\
Furniture & <Object> & Pyrolysis & <Process> & Action \\
Inner Environmental Field & $<$ Object> & Heat Transfer & <Process> & Action \\
Outer Environmental Field & <Object> & Heat Transfer & <Process> & Join \\
\hline
\end{tabular}

Table 1. Typical semantic interactions between the changes in indoor fire disaster

\subsection{Fire Simulation}

Fire Dynamics Simulator(FDS) is adopted in this paper to simulate a room fire development and Smokeview is used to display the results of an FDS simulation(McGrattan et al., 2008). The typical events like ignition and flashover can be recorded from the simulation results (Figure 5).

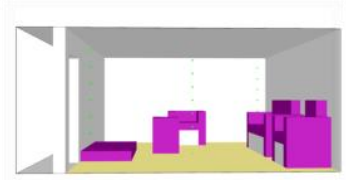

$\checkmark$ Room Scene

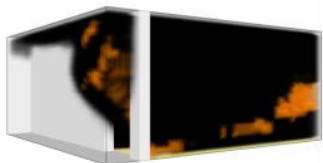

$\checkmark$ Flashover (Flame and Smoke)

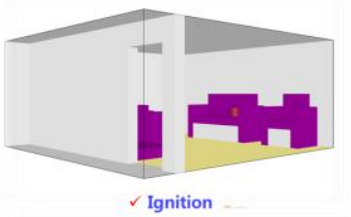

$\checkmark$ Ignition

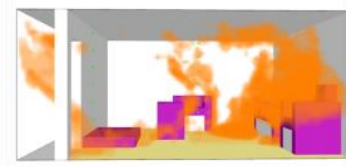

$\checkmark$ Flashover (Flame)
Figure 5. The simulation results from FDS for validation of ignition and flashover events

After RGIS monitors the event that the sprinkle begins to spray, its behavior will affect the heat transfer process which changes the simulation trend as shown in Figure 6. At the 2.0s after the ignition, the upper temperature of flame can reach $300^{\circ} \mathrm{C}$ but the water particles from the sprinkle will lower the temperature to up to $90^{\circ} \mathrm{C}$.

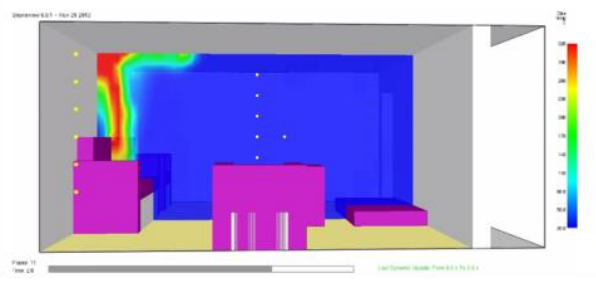

$\checkmark$ Before the sprinkle works

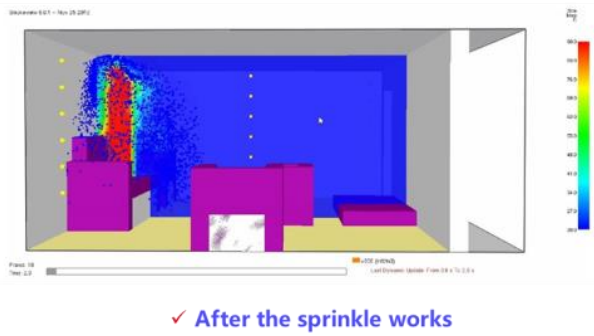

Figure 6. The simulation results from FDS for validation of sprinkle-spray event
According to the real-time data about the environmental field, the fire simulation model can be further optimized to adapt to the fire scene, and the evacuation network will also be updated which will be researched in future work. When more information closer to real-world changes can be obtained from RGIS, the decision support will be made more practical.

\section{ACKNOWLEDGEMENTS}

The research for this paper was funded by the National High Technology Research and Development Program of China (No. 2012AA121401 and 2013AA122301), and the National Basic Research Program of China (973 Program, No. 2011CB302306).

\section{REFERENCE}

Aggarwal, C., 2011. An Introduction to Social Network Data Analytics. In Social Network Data Analytics, ed. C. C. Aggarwal. Springer US, pp.1-15.

Artz, M., 2013. The New Age of Real-Time GIS. ESRI. http://blogs.esri.com/esri/esri-insider/2013/04/01/thenew-age-of-real-time-gis/ (1 May, 2013).

Claramunt, C.,Jiang, B.,2000. Hierarchical reasoning in time and space. In Proceedings of 9th International Symposium on Spatial Data Handling, 41-51.

Claramunt, C., Parent, C.,Thériault, M., 1998. Design Patterns for Spatio-temporal Processes. In Data Mining and Reverse Engineering, eds. S. Spaccapietra \& F. Maryanski. Springer US, pp.455-475.

Claramunt, C.,Thériault, M.,1995. Managing Time in GIS: An Event-Oriented Approach. In Proceedings of the International Workshop on Temporal Databases: Recent Advances in Temporal Databases, 23-42. Springer-Verlag.

Collins, J. P., 2010. Sailing on an Ocean of 0s and 1s. Science, 327, pp.1455-1456.

ESRI. 2010. Tracking Server 10 (White Paper). www.esri.com/library/whitepapers/pdfs/trackingserver-10.pdf (1 May, 2013).

ESRI. 2013. ArcGIS Enables Real-Time GIS. http://www.esri.com/esrinews/arcnews/spring13articles/arcgis-enables-realtime-gis (May 1, 2013).

Goodchild, M. F., (2011) Looking Forward: Five Thoughts on the Future of GIS. ArcWatch. http://www.esri.com/news/arcwatch/0211/future-ofgis.html (1 May, 2013). 
Grenon, P.,Smith, B., 2004. SNAP and SPAN: Towards dynamic spatial ontology. Spatial cognition and computation, 4, pp.69-104.

Hey, A. J., Tansley, S.,Tolle, K. M., 2009. The fourth paradigm: data-intensive scientific discovery.

Hottel, H. C., 1984. Stimulation of Fire Research in the United States After 1940 (A Historical Account). Combustion Science and Technology, 39, pp.1-10.

Isikdag, U., Underwood, J.,Aouad, G., 2008. An investigation into the applicability of building information models in geospatial environment in support of site selection and fire response management processes. Advanced Engineering Informatics, 22, pp.504-519.

Karlsson, B.,Quintiere, J. G., 2000. Enclosure fire dynamics. CRC PressI Llc.

Kwan, M.-P.,Lee, J., 2005. Emergency response after 9/11: the potential of real-time 3D GIS for quick emergency response in micro-spatial environments. Computers, Environment and Urban Systems, 29, pp.93-113.

Langran, G.,Chrisman, N. R., 1988. A framework for temporal geographic information. Cartographica: The International Journal for Geographic Information and Geovisualization, 25, pp.1-14.

Laurini, R., Servigne, S.,Noel, G., 2005. Soft Real-Time GIS for Disaster Monitoring. In Geo-information for Disaster Management, eds. P. Oosterom, S. Zlatanova \& E. Fendel. Springer Berlin Heidelberg, pp.465-479.

Li, D., 2009. An Overview of Earth Observation and Geospatial Information Service. In Geospatial Technology for Earth Observation, eds. D. Li, J. Shan \& J. Gong. Springer US, pp.1-25.

McGrattan, K.,Forney, G. P., 2008. Fire Dynamics Simulator (Version 5), User's Guide. NIST special publication, 1019, pp.1-186.

Pelekis, N., Theodoulidis, B., Kopanakis, I.,Theodoridis, Y., 2004. Literature review of spatio-temporal database models. The Knowledge Engineering Review, 19, pp.235-274.

Peuquet, D. J.,Duan, N., 1995. An event-based spatiotemporal data model (ESTDM) for temporal analysis of geographical data. International journal of geographical information systems, 9, pp.7-24.

Rüppel, U., Abolghasemzadeh, P.,Stübbe, K.,2010. BIM-based immersive indoor graph networks for emergency situations in buildings. In Proceedings of the International Conference on Computing in Civil and Building Engineering, 65. University of Nottingham Press, Paper.

Reitsma, F.,Albrecht, J., 2005. Implementing a new data model for simulating processes. International Journal of Geographical Information Science, 19, pp.1073-1090.

Töreyin, B. U., Dedeoğlu, Y., Güdükbay, U.,Cetin, A. E., 2006. Computer vision based method for real-time fire and flame detection. Pattern recognition letters, 27, pp.49-58.

Washington, W. M., Buja, L.,Craig, A., 2009. The computational future for climate and Earth system models: on the path to petaflop and beyond. Philosophical Transactions of the Royal Society A: Mathematical, Physical and Engineering Sciences, 367, pp.833-846.

Worboys, M., 2005. Event - oriented approaches to geographic phenomena. International Journal of Geographical Information Science, 19, pp.1-28.
Yuan, M., 1999. Use of a Three - Domain Repesentation to Enhance GIS Support for Complex Spatiotemporal Queries. Transactions in GIS, 3, pp.137-159.

Yuan. 2001. Representing Complex Geographic Phenomena in GIS. Cartography and Geographic Information Science, 28, pp.83-96.

Yuan, M.,McIntosh, J., 2003. GIS representation for visualizing and mining geographic dynamics. Transactions in GIS, 2, pp.3-10. 